\section{Easy insertion of multiple posts}

FILPOST is the only prefabricated post system that can be customised to suit the restoration for root post and core build up. It can be bent and shortened without risk of fracture, enabling easy insertion of multiple posts into converging canals. It is engineered to be easier to place, even in difficult cases, in a faster, safer manner. There is more preserving of healthy tooth structure and it is stronger in use via its unique passive 'interlocking' system. It is $99.8 \%$ titanium, biocompatible and compatible with all dental materials. No drilling is required during placement thus avoiding risk of perforation. Its anatomical shape minimises dentine removal. Retention grooves along the post, working together with retention grooves formed within the canal surface, by the special Universal Groover, create a unique passive interlock that strengthens retention.

The self-threading, self-aligning FILPIN speeds and eases placement for self-shearing first time, every time once optimum depth is reached. After insertion, FILPIN can be easily bent to suit the restoration without breaking it or the tooth. Its unique thread design maximises retention strength without causing internal stresses that may lead to cracking or crazing. Filpin enhances retention of all types of restorations to dentine.

Available from your dental dealer. Email info@filhol.com; www.filhol.com.

\title{
Recommended by professionals, preferred by patients
}

In April Philips announced the planned launch of the Philips Sonicare ProtectiveClean toothbrush at the British Dental Conference and Dentistry Show this month. The new range incorporates Sonicare's patented technology - 31,000 brush strokes per minute creates dynamic fluid action which drives fluid forces deep into the interproximal spaces and along the gum line, delivering users a gentle, yet effective clean for healthier gums.

Designed to make good oral health more accessible, the striking ProtectiveClean range has options to suit different price points and includes important features recommended by dental professionals and preferred by patients. Primary among then is a unique pressure sensor technology which alerts users when they are applying too much pressure. A two-minute timer helps patients brush longer. A brush head replacement reminder tracks the time and pressure a brush head has been used for alerting patients exactly when to replace their brush head, ensuring the toothbrush is always performing at peak performance.
To support the launch, Philips is working with industry experts, Dr Ben Atkins, Clinical Director of Revive Dental and Anna Middleton, London Hygienist, to collect patient feedback and experience of using the New Philips Sonicare ProtectiveClean. Collating user feedback and results from more than 50 previous manual toothbrush users over a 4-week period, Dr Ben Atkins and Anna Middleton will present their results at the British Dental Conference and Dentistry Show this month.

The Philips Sonicare ProtectiveClean toothbrush is proven to reduce gum disease by up to $100 \%$ and remove up to seven times more plaque than a manual toothbrush for improved oral health. The toothbrush uses unique technology and a sonic sweeping motion to create gentle microbubbles for an exceptional clean feeling. Visit Philips

at the British Dental Conference and Dentistry Show to learn more about the Philips Sonicare ProtectiveClean 4300 and the Philips Sonicare ProtectiveClean 6100.

For more information, visit http://www.philips.co.uk/sonicare.

\section{Providing fantastic whitening value}

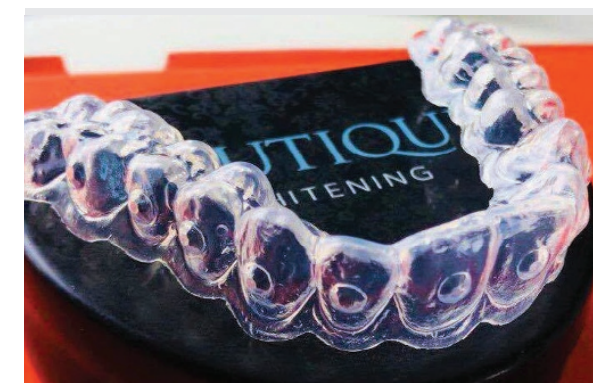

S4S Directors, Matt Everatt and Neil Bullement, are collaborating with Prem Sehmi, Owner of Boutique Whitening, to become the exclusive manufacturer of whitening trays for his company.
Boutique has become one of the UK's biggest providers of tooth whitening. Coupled with the S4S signature 'super-seal' trays, it is an unbeatable combination.

S4S are one of the UK's largest manufacturers of splints in the UK, providing work to over 6,000 dentists. The lab has gone from strength to strength in recent years, built on a reputation of uncompromising quality and impeccable customer service. Working alongside Boutique will create a whitening system that is unrivalled for quality, clinical efficiency and value.

There is emerging research by Mintel (2018) showing that $27 \%$ of UK adults are interested in having their teeth whitened.
Whitening is one of the least invasive and simplest ways to transform a patient's smile. There has never been a better time to offer Boutique Whitening in your practice.

The price of Boutique Whitening trays will remain unchanged, and any customers that have purchased whitening tray vouchers can use them as normal.

The hallmark of this partnership is to provide premium quality at fantastic value, and, with these two giants of the dental industry working together, for dentists and patients, the future is certainly bright.

Visit s4sdental.co.uk and boutiquewhitening.com. 